\title{
Exploring Satisfaction Towards Web Text Reading Among Dyslexic and Normal Learners
}

\author{
Chwen Jen Chen', Melissa Wei Yin Keong ${ }^{1}$, Chee Siong Teh ${ }^{1}$, and Kee Man Chuah ${ }^{2}$ \\ 'Faculty of Cognitive Sciences and Human Development, Universiti Malaysia Sarawak, Kota Samarahan, Malaysia \\ ${ }^{2}$ Centre for Language Studies, Universiti Malaysia Sarawak, Kota Samarahan, Malaysia \\ Email: \{cjchen, csteh\}@fcs.unimas.my, lissakeong@hotmail.com, kmchuah@cls.unimas.my
}

\begin{abstract}
The study aims to investigate the difference in satisfaction levels between normal and dyslexic learners when presented with various web text modes. Due to the high percentage of web users who exhibit some traits of dyslexia, presenting a web text that follows the guidelines which are meant for normal users poses challenges to dyslexic users. Acknowledging the ubiquitous use of the web for learning and the massive availability of text on the web as well as the significant number of dyslexic learners, this investigation intends to derive appropriate guidelines for displaying web text that could accommodate both normal and dyslexic learners. This qualitative study employs a multiple case study design and data are mainly collected via observation and guided interviews. The study reveals that existing dyslexia-friendly text guidelines are also appropriate for normal learners and contrary to the popular belief that assistive technology such as screen readers are helpful in reading, reading text aloud does not always work for both dyslexic and normal learners.
\end{abstract}

Index Terms - web text reading, inclusive guidelines, screen reader, dyslexia-friendly

\section{INTRODUCTION}

The definition and concept of dyslexia can be described in many ways. Some are based on medical models, while others are centered on the educational impact and the possible causes of dyslexia [1]. Literally, the word dyslexia is derived from Greek words, 'Dys' means poor or inadequate and 'Lexis' means words or language [2], [3]. It is more comprehensively defined as a specific learning disability which affects the development of literacy and language related skills which can hinder a person's ability to perform language-related tasks such as word recognition, reading, writing, spelling, reading comprehension and sometimes speaking [4], [5]. Dyslexia is often misconstrued as a person with poor intelligence, sluggishness or a result of impaired vision [6]. However, [7] and [8] stated that many people with this disability have average or above average intelligence and generally not linked to low intelligence.

To date, a number of guidelines are recommended to facilitate reading among people with dyslexia. For example, considering the visual stress experienced by dyslexics, [9] publishes a set of guidelines for creating

Manuscript received March 4, 2015; revised May 30, 2015. dyslexia friendly text and similar guidelines are also published by [10].

Numerous web text guidelines to cater the needs of normal users are also available, for example those by [11] and [12]. There are also inclusive recommendations on web text formatting that move beyond typical users to include users of all ages, experience levels, and physical or sensory limitations such as those proposed by [13] and Web Content Accessibility Guidelines (WCAG) that provide technical standards on how to make web content more accessible to people with disabilities [14]. According to [15], most of such inclusive recommendations consider diverse group of physical and cognitive disabilities instead of focusing on the specific needs of people with dyslexia.

Existing guidelines for web text accessibility focus either solely for dyslexia, normal or diverse (normal and all other types of disabilities and differences) web users. Minimal effort is known on deriving inclusive web text accessibility guidelines that are appropriate only for normal people and people with dyslexia. This study aims to yield guidelines that afford web text reading for both dyslexic and normal learners by exploring their satisfaction toward different web text modes.

\section{MEthodOLOGY}

This qualitative study employed a multiple case study design. It involved twelve dyslexic secondary school students ( 7 female, 5 male) as well as 12 normal secondary school students ( 8 female, 4 male), with their ages ranging from 14 to 18 years old. Data were collected by observing participants' behavior and their facial expression when using each of the web text modes as well as via guided interview sessions.

\section{A. Web Text Modes}

This study involved the use of three web text modes, named as Control, Standard and Enhanced. Each mode consisted of a reading passage. Table I shows the differences and similarities between these modes. In the Control mode, the passage was presented using the layout and typefaces that are similar to those commonly found in a conventional printed book. As for the Standard mode, the passage was presented based on some dyslexiafriendly text guidelines as suggested by [9]. The Enhanced mode was similar to the Standard mode except 